\title{
Os Cursos de Extensão em Saúde e Etnia na Faculdade de Saúde Pública da USP, ou a Diferença que Precisa Fazer Diferença
}

Extension Summer Courses on Ethnic Groups and Health at School of Public Health of USP, or the Difference that needs to make a Difference

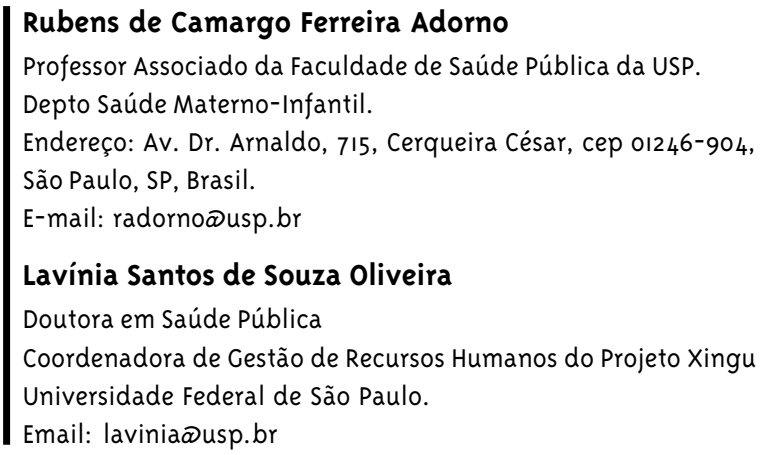

\section{Resumo}

Realiza-se uma breve discussão da introdução das noções de raça/etnia e etnicidade no campo da saúde pública no Brasil, destacando-se a emergência dessa questão no contexto da universalização das políticas públicas sociais e tendo como pano de fundo a experiência de realização de cursos de extensão na área de saúde pública.

Palavras-chave: Etnia e saúde; Recursos humanos em saúde; Saúde pública. 


\section{Abstract}

This report discusses the introduction of the conceps of race, ethnics and ethnicity in Brazilian public health policies highlighting the relationships between ethnicity and universal social policies, departing from universities extension courses in public health.

Keywords: Ethnic Group and Health; Health Human Resources; Public Health.

\section{Introdução}

O tratamento do tema de Etnia e Saúde fez parte do conteúdo de programas de extensão oferecidos nos cursos de verão da Faculdade de Saúde Pública da USP nos anos de 2002, 2003 e 2004.

A temática da raça/etnia, tratada como uma variável a ser estudada nas características da população, ou a partir de um estudo demográfico de uma população em particular, se colocara de maneira dispersa dentro dessa instituição de ensino, expressando-se também de uma forma menos crítica e reflexiva, sem incorporar uma discussão que fosse contextualizada historicamente.

A partir do ano 200o, a Faculdade de Saúde Pública da USP acumulou uma produção acadêmica de teses que procuraram contextualizar a discussão do tema, apontado como uma questão crítica, a ser reconhecida e problematizada tanto em relação à diferença entre perfis de mortalidade como em termos de problemas de acesso aos serviços de saúde por parte das populações, quando analisadas do ponto de vista das classificações contidas no censo demográfico do IBGE (Adorno e col , 2004).

O simples reconhecimento da diversidade étnica da população brasileira não trouxe consigo a necessidade de refletir sobre políticas voltadas para esses aspectos como diferenciadores das ações do Estado no sentido de busca de incorporação dentro de uma perspectiva mais ampla de cidadania. Pelo contrário, o reconhecimento da diversidade étnica ou racial em termos institucionais, sempre esteve voltado para a legitimação de uma ordem social de um passado escravocrata, que sob o rótulo de uma democracia racial, velava as características étnicas, ou a mestiçagem da população brasileira como categorias subsumidas às condições de dominação de classe nessa sociedade.

Nas últimas décadas, seja a partir da ação de movimentos sociais, ou da agenda de organizações internacionais ou multilaterais, essa questão começou a ser mobilizada. Como reflete Gilliam (200o), a conjuntura que no Brasil e na América Latina ficou conhecida como de ajuste "neo-liberal" colocou esse tema em termos de transformações do Estado com respeito a perspectiva mais ampla de cidadania. 
Destaca-se já como particularidade do caso brasileiro a consolidação de um Sistema Único de Saúde SUS - aparentemente no sentido contrário das medidas propostas em tais políticas de ajuste, como ocorreria em outros países da América Latina, com políticas de privatização da saúde e da previdência e adoção de um pacote mínimo de saúde.

Pensando no fato de que a adoção de um sistema universal de saúde se deu a partir de mecanismos de racionalização do Estado em relação às políticas anteriores - baseadas em um recorte corporativo como os IAPs (Institutos de Aposentadorias e Pensões) e pela divisão de funções entre o Ministério da Saúde e o Ministério da Assistência Social, bem como por uma agenda política colocada pela chamada redemocratização a partir da Constituição de 1988, o tema da saúde constitui-se, desde então, em um esboço de ampliação da cidadania.

Apesar da inscrição na agenda social das questões das etnias indígenas brasileiras e de populações tradicionais como quilombolas, que naquela conjuntura citada seriam inseridas por força de suas vinculações ao reconhecimento das terras de propriedade do Estado, verifica-se que as questões étnicas ou outras diferenças históricas e estruturais em relação à população brasileira não foram suficientemente mobilizadas tendo em vista um processo mais amplo de mudança no significado de cidadania.

O que se pode concluir é que a própria implantação do Sistema Único de Saúde quando analisado na perspectiva crítica da Saúde Pública/ Saúde Coletiva vem trazendo para a arena de discussão temas pertinentes à execução de direitos de cidadania, que, embora vistos certas vezes pelo viés da ampliação do acesso, ou da realização da eqüidade em saúde, vem mobilizando a discussão de temas que dizem respeito à própria configuração da relação entre Estado e sociedade, nos marcos do reconhecimento da nação. Assim os temas raça/etnia passam a ser visíveis, pois, envolveram historicamente e continuam a envolver de uma forma mais acentuada em um processo de profunda aceleração da internacionalização do capital os limites institucionais do Estado - Nação confrontados com as questões de origem das populações.

Do ponto de vista dos princípios que orientam as políticas do Sistema Único de Saúde: integralidade, eqüidade, participação e universalidade, a temática das inclusões torna-se um desafio para aprimoramento da assistência em um país marcado por grandes diferenças na distribuição de renda, com o funcionamento de um mercado de planos privados de saúde que procuram viabilizar-se como um diferencial de classes no atendimento, ficando o sistema público voltado aos pobres e "carentes". A classe média e setores mais especializados dos trabalhadores industriais e de serviços urbanos procuram viabilizar, a partir de um discurso político inclusivo no lema de suas organizações sindicais, a defesa de que um atendimento de qualidade a partir de um plano privado. (Adorno, 1992).

Não se pode esquecer, por outro lado, que uma das possibilidades dentro do espectro de governabilidade e de ação do Estado em um contexto de internacionalização do capital, que é o de atender setores ou estabelecer políticas focalizadas. Estas políticas podem estar sustentando no dia a dia um discurso de ampliação das cidadanias e dos direitos, ao mesmo tempo em que se convive com a tradição de um Estado que hierarquiza e privatiza a inserção da população.

Assim, observa-se que, também em uma agenda internacional, registraram-se tendências de um discurso de valorização das culturas não ocidentais em todo mundo, expressas nas diretrizes emitidas por organizações como a ONU, OIT, Unesco, e que procuram influenciar no conjunto de leis de cada país. Tais agendas sociais vêm dando relevância ao tema da eqüidade em saúde; nesse aspecto, corrigir assimetrias de acesso a serviços essenciais, buscar a melhoria de indicadores de saúde, adotar políticas de inclusão social de grupos populacionais minoritários e incrementar seus mecanismos de representação política, são fatos que podem ser considerados como novos e relevantes no panorama social (Oliveira, 2002).

As disparidades sociais são uma tônica na maioria dos países da América Latina e as políticas setoriais propostas pelos países ricos equacionadas dentro de modelos de metas e planejamento consoantes com as modalidades de avaliação das metas de investimento de capitais destacam, a partir de indicadores pré-estabelecidos, o combate à pobreza como prioridade. 0 mote do combate à pobreza nos países pobres, em desenvolvimento, ou nos mercados emergentes, busca focalizar em certa medida o controle das populações dessa parte do mundo, para evitar o seu afluxo nas áreas que acumularam a maior parte das riquezas. 
Dentro desse movimento observaram-se esforços de alguns órgãos como a OPAS/OMS e universidades no continente americano que vêm buscando descrever e compreender as relações entre saúde e etnia, enquanto constituintes dos diferenciais de saúde dentre os indivíduos. Partiu-se da idéia de que esse enfoque possa propiciar o delineamento e a problematização de um novo quadro demográfico, cultural e de saúde, possibilitando a emergência de novas categorias de análise e construção política.

Esse cenário estimulou na academia debates teóricos que transitaram pelas noções de raça, etnia e etnicidade e as fontes de informação para esse tipo de abordagem consideraram os descritores de saúde, o padrão de auto-referência na construção da identidade étnica e as influências culturais no estado de saúde das populações. Tornou-se consenso a necessidade de desconstruir o conceito de raça, considerando-o um constructo histórico de natureza muito mais ideológica do que científico e discriminante. Já o termo etnia evoca fatores de ordem cultural e social que baseiam ou pretendem visualizar a existência de um povo, ou de uma origem particular. A etnicidade apresentou-se como uma noção que encerra uma dimensão histórica e vincula-se intimamente aos problemas de classificação das pessoas e suas relações grupais. O que vem se chamando etnicidade se pretende uma noção dinâmica que se constrói a partir dos mitos, ancestralidade, origens comuns, território, memória de um passado coletivo, vestimentas, linguagem a ainda traços físicos (Poutignat e Streiff-Fenart, 1998 ) .

Esse debate se coloca, também, na perspectiva do que vem sendo chamada de globalização, que corresponde a um constructo cultural da intensificação das trocas de bens e capital entre os países. Etnia e identidades transitam não apenas como um dos produtos do mercado de consumo cultural, mas como aspecto que expressa as tensões produzidas por esse processo, entre standartização e culturas locais.

Santos (2001) ao discutir limites e possibilidades dos direitos humanos no processo de globalização destaca o que chama de "globalismos localizados" em oposição a "localismo globalizado". O primeiro corresponde a grupos que sob o impacto da globalização aprofundam seu processo de desestruturação e acabam por aumentar a desvalorização de grupos que acabam aprofundando sua submissão e condição de dis- criminação étnica; no segundo caso encontram-se aspectos ou manifestações locais que se incorporam como bens de valor no mercado global. Assim sendo, ao considerarmos o destaque que a globalização pode dar a etnicidade, esse aspecto pode tornar-se uma moeda de troca tanto de agregação de valor como de aprofundamento de sua condição de subordinação social. Destarte, deve-se pensar que o recorte pelo lado da etnia pressupõe a consideração do contexto sóciohistórico das populações e as articulações locais/nacionais/globais de cada grupo, e não apenas um recurso classificatório.

$\mathrm{Na}$ área específica da saúde, o foco sobre as características de origens histórico-sociais das populações, o que vem sendo chamado de relação entre saúde e etnia permite visualizar diferenciais no perfil epidemiológico dos povos do Brasil. Apesar de escassos, registros sistemáticos de saúde estão possibilitando o conhecimento do perfil de morbi-mortalidade de negros e indígenas brasileiros. A perspectiva étnica num apanhado demográfico evidencia que há sub-registros das populações indígenas e afro-descendentes, e são grandes as lacunas sobre informações de saúde. Ainda assim, são evidentes as taxas de mortalidade geral mais altas na população negra e indígena, altos índices de mortalidade infantil dentre os indígenas especialmente em áreas isoladas e grandes dificuldades de acesso aos serviços de saúde para diagnóstico e tratamento de doenças crônicas como a hipertensão entre os negros (OPAS, 2001). Merecem destaque ainda a qualidade desses serviços e o peso da segregação e discriminação praticadas nos próprios serviços.

Considerando esses aspectos é que se organizaram os eventos e os cursos na Faculdade de Saúde Pública, tomando-se como norte uma necessidade dos diferentes grupos da população poderem ter acesso e conhecimento de seus diferenciais de saúde. A questão da saúde se coloca não como um elemento específico ou característico de um grupo populacional delimitado, mas como expressão das condições históricas e sociais que determinaram um lugar social para cada grupo, a partir de suas características e possibilidades históricas. Nesse sentido, os diferenciais de saúde tornam-se questões concretas e reais.

Do ponto de vista da questão das populações indígenas, registram-se os avanços da especificidade indígena no bojo da Reforma Sanitária brasileira. São 
marcos a I Conferência Nacional de Saúde Indígena, realizada dentro da VIII Conferência Nacional de Saúde em 1986, a Constituição de 1988 que garante o direito à diversidade cultural e a cidadania indígena, a II Conferência Nacional de Saúde Indígena realizada em 1993, com maciça participação indígena e o estabelecimento em 1999 de um sub-sistema de atenção de saúde indígena no SUS, garantido pela Lei 9836, na forma de distritos sanitários.

Conhecer e acompanhar a evolução da Política de Saúde Indígena em seus mais variados matizes, seja dos aspectos antropológicos, epidemiológicos, de gestão e formação de recursos humanos, inclusive indígenas, vem ocupando alguns pesquisadores e acadêmicos, com a produção de trabalhos bastante interessantes e que podem trazer um novo olhar sobre a saúde pública como um todo.

Em 2001 foram realizados na FSP/USP dois seminários e várias reuniões de estudo, envolvendo a participação de 150 pessoas, dentre profissionais de saúde, acadêmicos e lideranças indígenas. Nos anos de 2002, 2003 e 2004 três edições de cursos de verão sobre a temática etnia e saúde, reuniram 62 participantes, inclusive indígenas.

Essa produção de material não se deu de forma sistematizada, ou a partir de um grupo ou núcleo que mantivesse essa preocupação, mas de maneira dispersa entre os diversos departamentos - o que demandava um esforço específico e uma limitação. Os cursos visaram implementar um diálogo e a existência do grupo saúde e etnia.

O presente trabalho visa apresentar algumas questões emergentes nos debates dos cursos de verão da FSP, descrever o perfil dos participantes e instituições e analisar as situações e repercussões trazidas para a saúde pública, a partir do conteúdo de relatórios e documentos relativos aos cursos de etnia e saúde.

Um primeiro aspecto refere-se ao título dos cursos que refletiram a cada ano de realização a tomada de discussão da temática a partir de um ponto de partida.

0 curso realizado no verão de 2001 chamava-se “ $A$ saúde indígena como ponto de partida para a questão etnia e saúde pública" e teve como temática central uma discussão das diferentes situações de vida das etnias indígenas no Brasil e os problemas e limites da montagem de uma política de atenção especial aos povos indígenas.
Os debates abrangeram os temas Etnia e Saúde como área emergente, Política de Saúde Indígena no contexto do SUS, Perfil epidemiológico das populações indígenas e sistema de informação em saúde indígena, Determinantes e condicionantes da saúde em populações indígenas, Relações entre diversidade cultural, produção de saúde e sistema público de saúde: o caso das DST/AIDS, Formação de Recursos Humanos para a área de saúde indígena: a formação de agentes de saúde indígena: Xingu, Maranhão e Bertioga.

Destaca-se a aula introdutória na qual a discussão sobre a noção de sabedoria como participante das sociedades indígenas e a noção de conhecimento prevalente nas sociedades ocidentais foi um ponto de partida para a discussão do que se determina como saúde. Durante o curso houve a participação de lideranças indígenas do estado de São Paulo e do Mato Grosso como palestrantes, finalizando o programa uma visita à aldeia Indígena Rio das Silveiras, localizada no município de Bertioga no litoral paulista.

O programa desenvolvido em janeiro de 2003 intitulou-se: "Etnia e Saúde: Perspectivas Brasileiras" e deu destaque à promoção do diálogo entre as áreas de saúde pública, educação, ciências sociais e antropologia sobre a questão da etnia e saúde. O objetivo principal foi de buscar subsídios para um pensamento brasileiro (bastante pretensioso) sobre produção social de saúde, redução de iniqüidades sociais e tratamento das diferenças entre os grupos humanos. 0 programa desenvolvido constou dos seguintes tópicos: Equidade em Saúde pelo prisma da etnicidade, Os índios: primeiros brasileiros,Tratamento Político das diferenças étnicas para populações indígenas e afrodescendentes, Relações entre etnia, pobreza e indicadores sociais de saúde no Brasil, Produção social da saúde :contribuições das culturas africanas e indígenas, finalizando com o Debate e apresentação cultural: Brasileiro que nem eu ? A construção da identidade brasileira dos imigrantes europeus e orientais.

A reflexão sobre diferentes origens da população e o recorte da linha da pobreza foram destaques dessa edição do programa, apresentado como painel para pensar a promoção e a assistência a saúde.

O curso de 2004 teve como título: "Etnia e diversidades em saúde: a construção de identidades no Continente" e seu programa teve pequenas variações em relação ao anterior, com o destaque de trazer a dis- 
cussão da proposta de inclusão do quesito cor/raça/ etnia nos registros de atendimento à saúde e de capacitar agentes do serviço de saúde a apresentarem essas questões no acolhimento a partir de uma perspectiva auto-classificatória.

\section{Participantes do curso e avaliação do conteúdo dos programas}

Nesses três programas desenvolvidos participaram 62 alunos. A graduação de origem dos participantes era variada, com maior participação de profissionais de enfermagem, $\mathbf{2 2} \%$; formados em ciências sociais, $\mathbf{2 0} \%$; $18 \%$ de médicos e $18 \%$ de dentistas, outras formações da área biológica: biólogos, nutricionistas e farmacêuticos representaram 10\% dos alunos. Professores, psicólogos, pedagogos, advogados, jornalista, arqueólogo e musicoterapeuta representaram os restantes $12 \%$ dos participantes .

Em relação às instituições a que pertenciam os profissionais destacaram-se os contratados pela FUNASA nas diversas aldeias indígenas do estado de São Paulo, FUNASA em aldeias e municípios do Estado do Amazonas, Centro de Referência Dsts/Aids São Paulo, Instituto da Criança do Hospital das Clínicas da USP, Secretaria de Estado da Saúde - São Paulo, profissionais contratados pela UNIFESP, Escola de Saúde Pública do Rio Grande do Sul, Prefeituras de municípios de Minas Gerais, Paraná e São Paulo e outros não vinculados a instituições públicas, participantes e não participantes de ONGs.

Na avaliação dos programas desenvolvidos os principais aspectos destacadas foram da seguinte natureza: - em relação à modalidade de curso: foi quase unânime a necessidade de cursos com maior número de horas que possibilitasse maior aprofundamento em temas considerados de relevância.

- em relação à temática: o alargamento do tema saúde etnia contemplando um espectro maior de questões e de grupos excluídos, assim como um aprofundamento das questões de discriminação. A integração do conteúdo do curso com a discussão de direitos humanos e a necessidade de aprofundar o conhecimento das populações indígenas no Brasil com informações sobre um número maior de etnias indígenas.

- em relação às contribuições do curso e a necessidade de sua realização foram destacados:
- necessidade de adquirir uma nova consciência étnica; complementação da formação de profissionais que têm uma formação eminentemente técnica sem refletir sobre as populações com as quais atuam, questão particularmente levantada pelos cirurgiões-dentistas que cursaram o programa;

- inclusão do tema diversidade na formação de quaisquer profissionais; e a necessidade de aprofundar a discussão sobre a formação de uma vontade política de interferir junto à população excluída para uma maior eqüidade, igualdade, construção de uma sociedade mais justa e mais igualitária.

Como questões gerais e de formação foi bastante destacado que o curso contribuiu para a realização de um projeto para diminuir as desigualdades e a formação de outros olhares, que levem a desnaturalizar a diferença.

Como sugestões para aprimorar os cursos ministrados foram registradas as seguintes respostas avaliativas:

- incluir a reflexão sobre outros grupos marginalizados; - trabalhar a relação com o meio ambiente;

- aproximar mais os antropólogos da área da saúde, incluir as questões ambientais junto com populações tradicionais: caiçaras, pantaneiros, quilombolas, caboclos; - trazer mais a questão da experiência que cada etnia tem e como concebe a saúde; conhecer mais sobre sistemas tradicionais de cura;

- conhecer mais sobre a cosmologia indígena.

\section{Discussão}

Após a avaliação e também o registro das discussões ocorridas durante os cursos pode-se concluir que em termos de uma direção geral da discussão proposta na área de saúde pública deve-se considerar que a vulnerabilidade das populações é conseqüência da violência estrutural que incide em países como o Brasil como um todo e de modo perverso sobre as comunidades mais pobres. Evidencia-se, mais uma vez, a natureza sócio-econômica das disparidades em saúde; a necessidade de identificar e registrar as diferenças entre as condições de vida e saúde dos grupos étnicos e a Invisibilidade dos dados no que se refere às populações rurais, negras e indígenas. 
Quanto à gestão e condução política de um sistema de atenção à saúde, destacam-se a fragilidade dos modelos de gestão pautados no recorte étnico, despreparo dos profissionais de saúde frente essa questão e dificuldades em se compor um campo de interdisciplinaridade.

A questão da etnia é prioridade em saúde pública. A possibilidade de conhecer e resgatar valores, bem como resgatar diferentes saberes e práticas de saúde, é enriquecedora. Essas diferenças na situação de saúde dos distintos grupos da população relacionam-se a fatores como sustentabilidade econômica e social e acesso a serviços de educação e saúde.

As Políticas de redução de pobreza, aliadas aos movimentos sociais e de direitos humanos, vêm constituindo dinâmicas interessantes. Há importantes lacunas no trato da especificidade da saúde evidenciadas pelos indicadores de morbi-mortalidade das populações indígenas e quilombolas que precisam ser trabalhadas com novas metodologias. Também ressalta-se a importância de conhecer e valorizar saberes e práticas tradicionais.

A experiência da oferta dos cursos na instituição foi extremamente válida, pois demonstrou que os alunos afirmaram a necessidade da formação em saúde incluir o reconhecimento das questões étnicas e sua legitimidade em termos da conquista de uma maior eqüidade em saúde. Também valorizaram a questão em termos de tratamento de direitos expressando-se inclusive na direção dos direitos humanos.

\section{Referências}

ADORNO, R. C. F.; ALVARENGA, A. T.;

VASCONCELLOS, M. P. Quesito cor no sistema de informação em saúde. Estudos Avançados, São

Paulo, v. 18, n. 5o, p. 119-123, 2004.

ADORNO, R. C. F. Movimento social e participação: a saúde na esfera pública. Saúde e Sociedade, São

Paulo, v.1, n. 2, p. 111-124, 1992.

GILLIAM, A. Globalização, identidade e os ataques à igualdade nos Estados Unidos: esboço de uma perspectiva para o Brasil. In: FELDMAN-BIANCO, B.; CAPINHA, G. (Org.). Identidades: estudos de cultura e poder. São Paulo: Hucitec, 20oo. p. 73-106.

OLIVEIRA, L. S. de S. Termo de referência para o momento indígena na III Conferência Regional de Promoção da Saúde. São Paulo: Union Internacional de Promocion de la salud y Educacion para la Salud. 2002.

OPAS - ORGANIZAÇÃO PAN-AMERICANA DA SAÚDE. Equidade em saúde: uma visão pelo prisma da etnicidade. Washington, DC, 2001. Disponível em:<http://www.paho.org/Spanish/HDP/hdd/ etnia.pdf $>$. Acesso em: 12 abr. 2007.

POUTIGNAT, P.; STREIFF-FENART, J. Teorias da etnicidade. São Paulo: Unesp, 1998.

SANTOS, B. de S. Globalização: fatalidade ou utopia. Porto, Edições Afrontamento, 2001. 